\title{
Prediction of Peaks in Wolf Numbers in Cycle 24 according to Actual Numbers of Polar Faculae
}

\author{
D. K. Callebaut ${ }^{1, *} \&$ V. V. Makarova ${ }^{2, * *}$ \\ ${ }^{1}$ Physics Department, CGB, University of Antwerp, B-2020 Antwerp, Belgium. \\ ${ }^{2}$ Kislovodsk Solar Station of the PAO, 357700 Kislovodsk, Russia. \\ *e-mail: Dirk.Callebaut@ua.ac.be \\ **e-mail:mahatt@rambler.ru
}

\begin{abstract}
Previous investigations by Makarov et al. have shown a relation between the peaks in the number of polar faculae and the peaks in the Wolf number. In cycles 20 and 22 the delay between peaks in polar faculae and Wolf number was $6.1 \pm 0.1$ year, north and south taken separately, as their peaks do not coincide. For the odd cycle 21, this shift was 5.6 years average. Polar faculae always precede the sunspots. The relevance of this for the dynamo mechanism is obvious. In cycle 23 the delay was 7.7 year (north) and 7.8 year (south). The approach of a deep minimum is probably responsible for this increased delay; thus for cycle 24 the delay between peaks of polar faculae and sunspots is expected to be at least 7.8 years and probably longer. The present polar faculae show 6 peaks above the smoothed average (north) and similarly 3 peaks south. The first peak for the sunspots will be at the earliest during the very end of 2007. As soon as one peak in the spots occurs the delay for cycle 24 can be estimated and the other peaks predicted.
\end{abstract}

Key words. Polar faculae—sunspots—dynamo—-Wolf number.

\section{Introduction}

During a long time polar faculae (PF) were considered as a phenomenon following the sunspots (SP) like a tail end. Several publications by Makarov and coworkers (Makarov et al. 1989, 2008; Makarov \& Makarova 1996) showed that the peaks in the number of PF preceded the peaks in the SP by about half a cycle. Makarov and Makarova (1996) found for cycles 19 to 21 an average correlation factor 0.84 between the PF and the SP following them ( $\mathrm{N}$ and $\mathrm{S}$ separate), while the correlation between $\mathrm{PF}$ and the preceding SP was still 0.72 on the average. However, the meaning of these results is relatively restricted as both phenomena (PF and SP) wax and wane with the same period and roughly in a similar way. That the correlation between the PF and the following SP is higher than with the SP preceding them is due to the peaks and thus the peaks were considered separately, giving a clear correlation between PF and the SP following them and a bad correlation between PF and the SP preceding them.

On the other hand, the relation between the total number of PF and the total number of sunspots does not seem to be simple as the total number of PF increased during the 
last 4 cycles by a factor larger than 3, while the number of SP decreased during the last cycle. We may interpret this situation at least partially as follows: the polar cap of the global magnetic field region increased during the last 12 cycles and this probably means that the region of $\mathrm{PF}$ is enlarged too, especially in the latitude band $35^{\circ}-55^{\circ}$ where they are better observable. However, this can only be part of the explanation. It may be noted that the present tendency of the number of PF is again decreasing (PF cycle 24 will give about half of cycle 23), which suits the general tendency of approaching a deep minimum. Moreover, according to the Greenwich Observatory (1880-1954), the high-activity PF cycles of 1887 and 1944 were followed by very strong sunspot cycles, and the weakest PF cycles in 1879 and 1901 preceded the weakest sunspot cycles. A relation between the polar magnetic field and the sunspot number in the next sunspot cycle has been used for predictions of future sunspot numbers (Schatten et al. 1978; Schatten 1986; Makarov 2002; Duhau 2003; Svalgaard 2005). This was taken into consideration by some authors to predict the next cycle, e.g., Choudhury et al. (2007).

\section{Cycles 20-23}

Makarov et al. (2008) give figures and a table showing that for cycles 20 and 22 the peaks in the PF precede the peaks in the SP by $6.1 \pm 0.1$ year. For the odd cycle 21 it was $5.7(\mathrm{~N})$ and $5.4(\mathrm{~S})$ year. The correlation factor varied from 0.53 to 0.63 . Peaks in the northern and southern hemisphere did not occur simultaneously but showed the same shift.

Figure 1( $\mathrm{a}$ and $\mathrm{b}$ ) for cycle 23 confirms the correlation between the PF and the SP which follow the PF. However, now the shift is much longer: $7.7(\mathrm{~N})$ and $7.8(\mathrm{~S})$ year (correlation factor $0.61(\mathrm{~N})$ and $0.71(\mathrm{~S})$ ). This contrasts even more with the short shift for cycle 21, i.e., the odd cycle clearly gave a shorter shift than the even cycles; in fact this suits the general feature that shorter PF cycles precede odd SP cycles (Makarov et al. 2003). The reason for the longer shift for cycle 23 is probably the approach of a deep minimum which according to Makarov et al. (2007) is expected to occur in cycle $26 \pm 1$.

Another difference for cycle 23 is that the peaks north and south occur nearly simultaneously: about a month difference, while for cycles 20-22 this was several months. However, cycle 23 shows asymmetry between north and south in other aspects (Zharkova et al. 2004; Georgieva et al. 2007).

\section{Cycle 24}

Figure 2( $a$ and $b$ ) represents the peaks of the PF, i.e., what remains after subtracting the smoothed average. The period is from 2001 till 2007, which is relevant for the $\mathrm{SP}$ in cycle 24. For the northern hemisphere we have 6 peaks (not counting the small ones), for the southern hemisphere we have 3 serious peaks. It is obvious that the $\mathrm{N}-\mathrm{S}$ symmetry which occurred in cycle 23 has now disappeared. Taking the same shift of 7.8 years as for cycle 23 then we may expect peaks in the SP in 2008.0, 2008.9-2009.1, 2011.0, 2011.6, 2012.1 and 2014.2 (north) and 2010.5, 2011.5-2011.9, 2014.3-2014.5 (south) approximately. However, this shift is uncertain and it is probably longer. In that sense it seems necessary to wait for the first peak (or first 2 peaks) in SP to determine the shift which can then be used to predict the following peaks. 

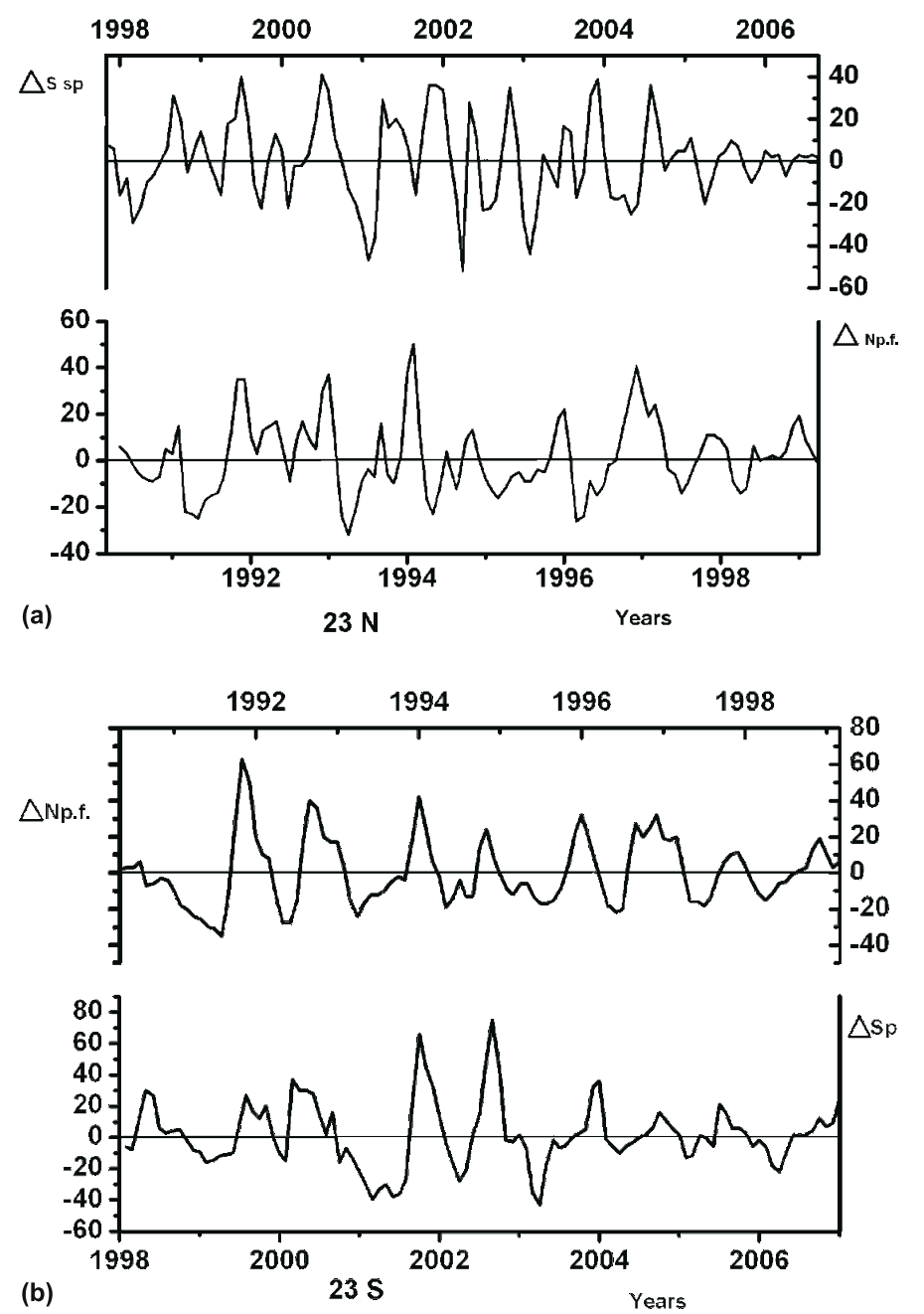

Figure 1. Cycle 23: the peaks in the number of polar faculae preceding the peaks in sunspots by 7.7 years (a) north and 7.8 years (b) south.

\section{Conclusion}

The fact that the PF precede the SP (at least the peaks do) has relevance for the BabcockLeighton theory and for the theories of Choudhuri, of Dikpati and of Callebaut \& Khater (2006). The latter theory has the advantage that it is based on an exact solution of the MHD equation and that PF and SP are generated naturally by the same mechanism, but its explanation (based on centrifugal forces) for the long delay of about half a cycle between PF and SP is now withdrawn. Instead, it is suggested that the meridional motion, on its return near the tachocline, releases first the PF and half a cycle later (and closer to the equator) the SP, which have been further amplified meanwhile and thus are stronger than the PF. This would solve the problem of the PF preceding the SP for all the theories mentioned above. The fact that the total number of PF per cycle is not 

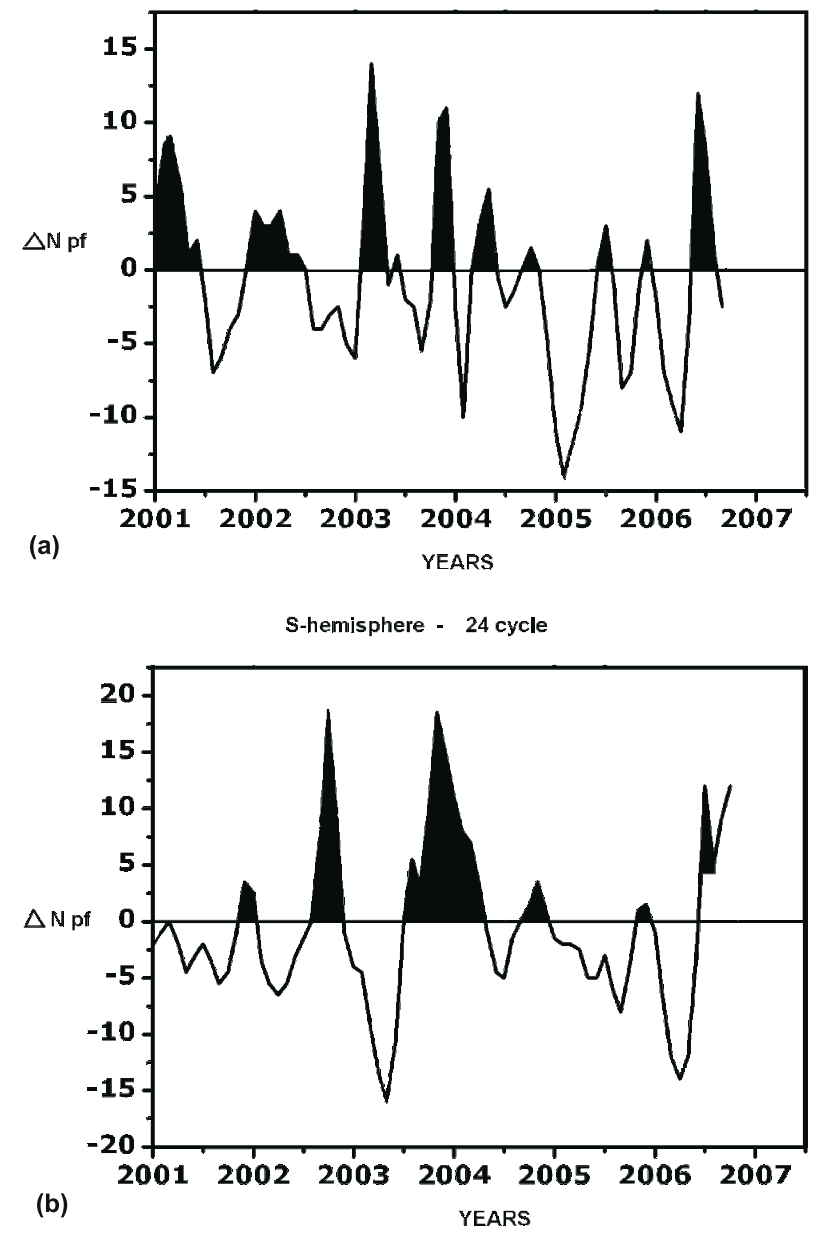

Figure 2. The peaks in the number of polar faculae (i.e., after subtracting the smoothed number of polar faculae): (a) north and (b) south. Period from 2001-2007, i.e., bearing on cycle 24 for the sunspots.

connected in a direct way with the total number of SP indicates that other effects have to be taken into account too.

\section{Acknowledgment}

One of us (V. V. M.) is grateful for the support of the Russian Fund of Basic Researches, project 05-02-16229 and the Program of the Russian Academy of Science.

\section{References}

Callebaut, D. K., Khater, A. H. 2006, In: Solar Activity and its Magnetic Origin (eds) Bothmer, V., Hady, A. A., page 9, Proc. IAUS 233, Cambridge Univ. Press. 
Choudhuri, A. R., Chatterjee, P., Jiang, J. 2007, arXiv:astro-ph, 0701527.

Dikpati, M. 2005, Adv. Space Res., 35, 322.

Dikpati, M., de Toma, G., Gilman, P. A. 2006, Geophys. Res. Lett., 33, L05102.

Duhau, S. 2003, Solar Phys., 213(1), 203.

Georgieva, K., Kirov, B., Tonev, P., Guineva, V., Atanasov, D. 2007, In: Sec. Internat. Symposium on Space Climate (eds) Mursula, K., Maris, G., Usoskin, I., AdvSpR, 40(7), 1152.

Makarov, V. I., Makarova, V. V., Sivaraman, K. R. 1989, Solar Phys., 119, 45.

Makarov, V. 2002, Private communication.

Makarov, V. I., Makarova, V. V. 1996, Solar Phys., 163, 267.

Makarov, V. I., Makarova, V. V., Callebaut, D. K. 2008, Solar Phys., in press.

Makarov, V. I., Tlatov. A. G., Sivaraman, K. R. 2003, Solar Phys., 214, 41.

Schatten, K. N., Scherrer, P. H., Svalgaard, L., Wilcox, J. M. 1978, Geophys. Res. Lett., 5, 411.

Schatten, K. N. 1986, In: Solar-Terr. Predict., (eds) Simon, P. A., Heckman, G., Shea, M. A., page 92, Proc. Workshop, Meudon, NOAA.

Svalgaard, L., Cliver, E. W., Kamide, Y. 2005, Geophys. Res. Lett., 32, L01104, doi:10.1029/2004GL021664.

Zharkova, V. V., Zharkov, S. I., Benkhalil, A. K. 2004, Mem. S. A. It, 75, 282. 